\title{
Clinical dosimetric impact of Acuros XB and analytical anisotropic algorithm (AAA) on real lung cancer treatment plans : review
}

\author{
Suresh Rana \\ Department of Medical Physics, ProCure Proton Therapy Center, Oklahoma City, Oklahoma, USA.
}

Received November 22, 2013; Revised January 15, 2014; Accepted January 16, 2014; Published Online January 17,2014

\section{Review Article}

\begin{abstract}
Photon dose calculation algorithms in treatment planning system could affect the accuracy of dose delivery when tissue heterogeneity is involved along the beam path. Treatment planning for lung cancer is challenging, especially in the case of treatment plan involving small fields. The combination of low-density (air) medium and small fields cause charge particle disequilibrium nears the air/tissue interface. Beam modeling within the dose calculation algorithms must also employ an accurate method of accounting tissue heterogeneity corrections in order to avoid dose overestimation or underestimation. Analytical anisotropic algorithm (AAA) is one of the widely tested and validated dose calculation algorithms in external beam photon radiation therapy. Recently, Acuros XB (AXB) was made available for photon dose calculations, and several studies have demonstrated better dose prediction accuracy of the AXB over AAA. This article reviews the results from the treatment planning studies, which have investigated the clinical dosimetric impact of the AXB and AAA on real lung cancer treatment plans.
\end{abstract}

Keywords: Acuros XB; Analytical Anisotropic Algorithm; Lung cancer; Tissue heterogeneity correction; Treatment planning

\section{Introduction}

Intensity modulated radiation therapy (IMRT) and volumetric modulated arc therapy (VMAT) are two most commonly used treatment delivery techniques in external beam photon radiation therapy. Both the IMRT and VMAT are capable of delivering conformal dose distributions to the target volume while minimizing dose to the organs at risk (OAR). ${ }^{1}$ One of the factors that could affect the accuracy of dose delivery in external beam radiation therapy is the dose calculation algorithm implemented within the treatment planning system (TPS). The treatment delivery to various tumor sites such as lung, breast, brain, oral cavity, and head and neck often involves the tissue heterogeneities, which have different radiologic properties. ${ }^{2}$ The Task Group 65 (TG-65) of the American Association of Physicists in Medicine (AAPM) states that "the general principle of $3 \%$ accuracy in dose delivery with the corresponding need for better than $2 \%$ accuracy in correcting for inhomogeneities is a reasonable, albeit challenging, goal."2

Corresponding author: Suresh Rana; Department of Medical Physics, ProCure Proton Therapy Center, Oklahoma City,

Oklahoma, USA. Email: suresh.rana@gmail.com

Cite this article as:

Rana S. Clinical dosimetric impact of Acuros XB and analytical anisotropic algorithm (AAA) on real lung cancer treatment plans: review. Int J Cancer Ther Oncol 2014; 2(1):02019.

DOI: $10.14319 / \mathrm{ijcto} 0201.9$
A number of studies have shown the limitation of photon dose calculation algorithms such as analytical anisotropic algorithm (AAA), collapsed cone convolution (CCC) algorithm, and pencil beam convolution $(\mathrm{PBC})$ algorithm when complex geometry with inhomogeneity is involved along the photon beam path. ${ }^{3-12}$ Both the AAA and CCC are based on the superposition/convolution method, which calculates the dose by superposition of dose kernels of primary and scatter components that are derived from the Monte Carlo (MC). The tissue inhomogeneity correction in superposition/convolution method such as in the AAA is done both in the beamlet direction and lateral directions. ${ }^{12-15}$ Specifically, the beamlet direction includes the radiologic scaling of the dose deposition functions, whereas the lateral directions include the electron-density-based scaling of photon scatter kernels. ${ }^{12-15}$ The inhomogeneity correction in the PBC, however, is limited since it does not consider secondary electron transport. $^{3}$

Recently, Varian's Eclipse TPS (Varian Medical Systems, Palo Alto, CA) has implemented Acuros XB (AXB) dose calculation algorithm, and it is commercially available for clinical use. The AXB is considered to be similar to MC, and it utilizes the linear Boltzmann transport equation (LBTE) and solves numerically that describes the macroscopic behavior of ionizing particles as they travel through and interact with mat- 
ter. ${ }^{16}$ Detail descriptions on the AXB can be found elsewhere. ${ }^{16-17}$

Current literature on the AXB shows that several investigators have validated the $\mathrm{AXB}$ by performing measurements and Monte Carlo (MC) simulations ${ }^{16-23}$ and reported better dose prediction accuracy using the AXB than using the AAA in heterogeneous media. If the $\mathrm{AXB}$ is more accurate than the $\mathrm{AAA}$, it is imperative to evaluate the clinical dosimetric impact of the AXB on real cancer treatment plans. The purpose of this article is to review the current literature on the clinical dosimetric evaluation of the AXB in lung cancer treatment plans and summarize the latest findings on this topic. The treatment planning for lung cancer is challenging, especially using stereotactic body radiation therapy (SBRT) technique, which involves low-density (air) medium and small fields causing charge particle disequilibrium near the air/tissue interface. ${ }^{24,} 25$ Moreover, both the calculated and measured doses have strong dependence on the irradiated inhomogeneous medium when electronic disequilibrium occurs. ${ }^{24}$ Therefore, beam modeling within the dose calculation algorithms must employ more accurate method of accounting tissue heterogeneity corrections in order to avoid dose overestimation or underestimation. However, due to the difference in the beam modeling approach, computed results of one dose calculation algorithm may not be in agreement with that of another dose calculation algorithm.

\section{Literature review}

In one of the most recent studies on the AXB, Liu et al. ${ }^{26}$ investigated the dosimetric impact of the AAA and AXB by performing dose calculations on the treatment plans of 77 lung cancer patients. It was reported that the AXB resulted lower conformation number $(0.650$ vs. 0.674$)$ and higher target dose heterogeneity ( 0.088 vs. 0.074$)$ when compared to the AAA. Similar result was reported in another study done on 16 SBRT lung cases. ${ }^{22}$ Specifically, the AXB plans produced lower plan conformity (0.77 vs. 0.82$)$ and higher target dose heterogeneity index (1.18 vs. 1.13$)$ in comparison to the AAA plans. ${ }^{22}$ Kathirvel et al. ${ }^{20}$ also reported lower conformity index in the AXB plans (1.1) than in the AAA plans (1.2) for 5 SBRT cases. The evaluation of the maximum PTV dose showed that the AXB plans produced smaller hot spot, with an averaged maximum PTV dose in the AXB plans higher by about $2.3 \%$ when compared to the AAA plans. ${ }^{22}$

Kroon et al. ${ }^{19}$ evaluated the AXB for stage I and stage III lung cancer patients. The findings from Kroon et al. ${ }^{19}$ showed lower near-minimum PTV dose in the AXB plans than in the AAA plans, with differences of up to $-12.3 \%$ for stage I ( $\mathrm{n}=$ 8 )and up to $-0.8 \%$ for stage III $(n=7)$ lung cancer plans. ${ }^{19}$ The mean PTV dose was also found to be lower in the AXB plans by up to $-4.9 \%$ than in the AAA plans. ${ }^{19}$ In agreement to their findings ${ }^{19}$, Rana et al. ${ }^{22}$ reported that the AXB produced lower mean and minimum PTV doses by $-0.3 \%$ and $-4.3 \%$, respectively, and Kathirvel et al. ${ }^{20}$ showed lower mean PTV dose in the AXB plans when compared to the one in the AAA plans for 5 SBRT cases (102.2\% vs. $102.9 \%$ of the prescription dose) as well as for 5 non-SBRT cases $(100.3 \%$ vs. $101.2 \%$ of the prescription dose). However, the AXB plans may not always produce lower mean PTV dose, which may depend on the photon beam energy and the location of the target. For instance, Fogliata et al. ${ }^{27}$ investigated the dosimetric impact of energy comparing 6 and $15 \mathrm{MV}$ photon beams, and their results showed that, in comparison to the AAA, the AXB predicted lower mean PTV doses in the soft tissue by up to $-1.7 \%$ for $6 \mathrm{MV}$ beam and up to $-1.2 \%$ for $15 \mathrm{MV}$. However, for the target in the lung, the mean PTV dose was higher by up to $1.2 \%$ for $6 \mathrm{MV}$ beam and lower by up to $2.0 \%$ for $15 \mathrm{MV}$ beam. ${ }^{27}$ Hence, the variations in the PTV doses among different studies may be attributed to the difference in the PTV volume, inclusion of lung/air volume inside the PTV, and location of the PTV with respect to the lung and chest wall.

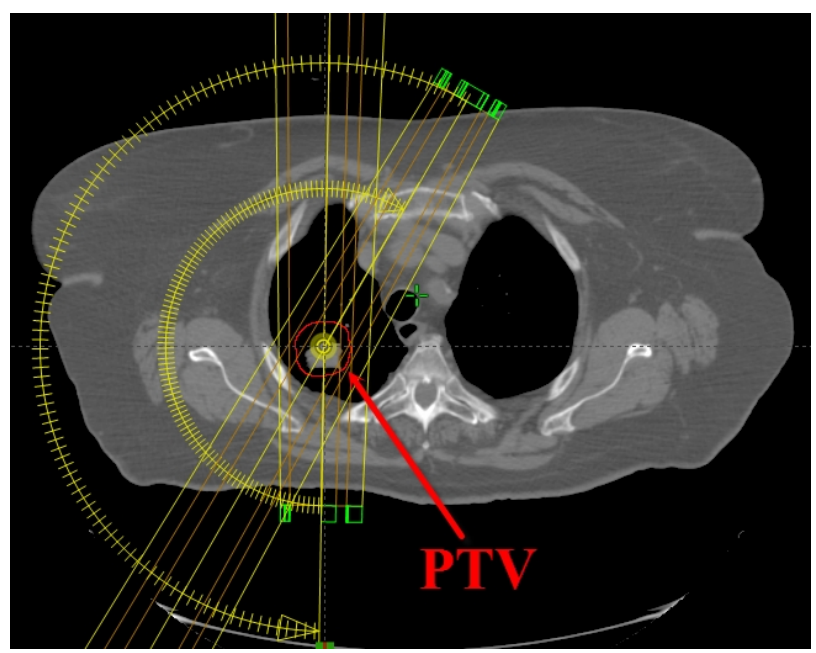

FIG. 1: A lung treatment plan setup using 2 partial arcs in Eclipse TPS. This figure shows the isocenter at the center of the planning target volume (PTV).

For the PTV, it is a standard practice to evaluate its coverage by calculating a certain percentage (e.g., 95\%) of the PTV volume covered by the prescription dose or certain percentage of the prescription dose. For the same number of monitor units (MUs), Rana et al. ${ }^{22}$ reported that the PTV coverage in the AXB plans was reduced by about $8 \%$ when compared to the one in the AAA plans. Such reduction in the PTV coverage using the AXB raises the question if the prescription dose needs to be adjusted for the AXB calculations in the lung plans in order to improve the target coverage. Based on the results of Rana et al. ${ }^{22}$, the MUs in the AXB plans had to be increased by $0.4 \%$ to $5.3 \%$ in order to obtain the same PTV coverage as in the AAA plans. Khan et al. ${ }^{28}$ investigated the effect of the AXB on MUs for 97 SBRT lung cases. The results from Khan et al. ${ }^{28}$ showed that the AXB required about $2 \%$ higher MUs than the AAA in order to deliver the prescribed dose. 
From the clinical perspective of using the AXB, it raises the question: can $A X B$ meet the dosimetric criteria of currently existing lung protocols? Recently, dosimetric evaluation of both the AXB and AAA was done based on the RTOG 0813 dosimetric parameters for 14 SBRT cases. ${ }^{29}$ The results from that study ${ }^{29}$ demonstrated that both the AXB and AAA could meet the RTOG 0813 dosimetric criteria for the SBRT lung, and the dosimetric values were lower in the AXB plans than in the AAA plans except for the normal lung tissue. For the lung, literature shows that the AXB produces slightly higher relative volume of the normal lung tissue receiving $20 \mathrm{~Gy}$ (V20) than the AAA. ${ }^{19}, 20,22,29$ Similarly, on average, higher dosimetric values were obtained in the AXB plans for the relative volume of normal lung receiving $5 \mathrm{~Gy}$ (V5). ${ }^{19,}{ }^{22}$ In contrast, the dosimetric values for both the spinal cord (maximum dose) and heart (maximum and mean dose) were found to be slightly lower in the AXB plans than in the AAA plans. $^{20,} 22$ However, there was no significant difference in the mean dose to lung between the AXB and AAA plans. ${ }^{19,20,22}$

\section{Discussion}

Previous experimental and MC studies comparing AXB vs. AAA have shown that the AXB is more accurate than the AAA in the presence of inhomogeneity. ${ }^{16-23,29-34}$ For instance, in MC study involving low-density lung, Bush et al. ${ }^{17}$ showed the difference between the MC and AAA was higher (up to $17.5 \%)$ when compared to the one between the MC and AXB (up to $4.5 \%$ ). In a phantom study involving low-density medium (lung insert and air gap), Rana et al. ${ }^{29}$ showed that the dose prediction error of the AAA could be up to $12.5 \%$ when compared to the measurements, whereas the AXB had better agreement with the measurements with a difference up to $2.8 \%$.

Although the AXB could improve the accuracy of dose calculations and avoid miscalculation of the MUs in the lung cancer treatment plans, it is not clear which dose reporting mode in AXB should be used for a clinical purpose. The AXB in the Eclipse TPS can report the absorbed dose in two modes: dose-to-medium $\left(\mathrm{D}_{\mathrm{m}}\right)$ and dose-to-water $\left(\mathrm{D}_{\mathrm{w}}\right)$. In recent years, there has been significant interest in using dose calculation algorithms that are based on MC approach, which can report the absorbed dose in $\mathrm{D}_{\mathrm{m}}$ mode. For the $\mathrm{AXB}$ in the Eclipse TPS, the default dose reporting mode is $D_{\mathrm{m}}$. The major difference between the AXB_Dm and AXB_Dw calculations comes from the post-processing step. Specifically, the radiation transport model in the AXB calculates the energy-dependent fluence, which gets multiplied by flux-to-dose response functions, and this gives the absorbed dose in the local voxel. ${ }^{16-17}$ Furthermore, for the AXB_Dm calculations, the macroscopic energy deposition cross-section and atomic density are based on the material properties of local voxel ${ }^{16-17}$; whereas, energy deposition cross-sections for water are used for the local media in the case of the AXB D $\mathrm{D}_{\mathrm{w}}$ calculations. ${ }^{16-17}$

A number of studies have investigated the difference between the $\mathrm{D}_{\mathrm{m}}$ and $\mathrm{D}_{\mathrm{w}}$ in AXB using MC simulations ${ }^{31}$ and phantom measurements. ${ }^{32,33,34}$ While the MC simulations and phantom measurements provide the dosimetric differences between the $A X B \_D_{m}$ and $A X B \_D_{w}$ calculations ${ }^{31-34}$, the literature comparing $\mathrm{D}_{\mathrm{m}}$ vs. $\mathrm{D}_{\mathrm{w}}$ on the real CT dataset of lung cancer patients is yet to be published. However, it is relevant to mention studies, which have investigated the dosimetric impact of the $\mathrm{D}_{\mathrm{m}}$ and $\mathrm{D}_{\mathrm{w}}$ on the real cases other than lung cancer. Kan et al. ${ }^{34}$ reported that, in comparison to the AXB_Dm, the AXB_Dw will calculate higher mean PTV doses by up to $4 \%$ and higher mean doses to the tissues adjacent to bone by up to about $2 \%$ for nasopharygeal carcinoma. Fogliata et al. ${ }^{35}$ reported that the dose distributions calculated by the $A X B \_D_{w}$ were about $5 \%$ higher than corresponding ones calculated by the AXB_Dm for soft-tissue sarcoma patients. Dogan et al. ${ }^{36}$ reported that conversion of $\mathrm{D}_{\mathrm{m}}$ to $\mathrm{D}_{\mathrm{w}}$ in $\mathrm{MC}$ method led dose discrepancies up to $8.0 \%$ for prostate cancer and up to $5.8 \%$ for head and neck cancer. These discrepancies between the $D_{m}$ and $D_{w}$ calculations may be due to the difference between stopping powers of water and materials of different densities.

Since the $D_{w}$ and $D_{m}$ in the $A X B$ can result different dosimetric results, it is essential to further investigate if the selection of either the $D_{m}$ or $D_{w}$ in AXB will make any significant difference for the lung plans in the clinical environment. Additionally, the dosimetric impact of the $D_{m}$ and $D_{w}$ in the AXB may also depend on the treatment techniques (e.g., IMRT, VMAT, and SBRT). The use of $D_{m}$ vs. $D_{w}$ for external beam photon radiation therapy still remains a debating topic. ${ }^{37}$ The supporters of the $\mathrm{D}_{\mathrm{m}}$ reason that (1) conversion from the $D_{m}$ to $D_{w}$ adds uncertainty in dose calculations due to uncertainties in computed stopping power ratios; (2) $\mathrm{D}_{\mathrm{m}}$ is more likely to provide a better measure of biological response; (3) changing to the $D_{m}$ will have minimal impact on the treatment protocols; and (4) conversion of the $D_{m}$ to $D_{w}$ defeats a potential advantage of using MC-based dose calculation algorithms. The supporters of the $D_{w}$ argue that (1) commissioning beam data are always measured in water; (2) dosimetry calibration protocols are based in water; (3) clinical experience in terms of tumor/tissue response is based on the $\mathrm{D}_{\mathrm{w}}$, and (4) conversion from the CT numbers to media results uncertainty in the medium type and composition.

Despite the ongoing debate on whether the $D_{w}$ or $D_{m}$ is the most appropriate to report the dose in external beam photon radiation therapy, the clinical outcome data comparing both the $D_{m}$ and $D_{w}$ remains to be reported. Nevertheless, the current literature supports the use of the AXB over the AAA for dose calculations, especially for the lung treatment plans. It is recommended that the $\mathrm{AXB}$ users consider the arguments ${ }^{37}$ 
$D_{m}$ vs. $D_{w}$ before implementing either the $A X B \_D_{m}$ or AXB_Dw for a routine clinical use.

\section{Conflict of interest}

The author declares that he has no conflicts of interest. The author alone is responsible for the content and writing of the paper.

\section{References}

1. Otto K. Volumetric modulated arc therapy: IMRT in a single gantry arc. Med Phys 2008; 35:310-317.

2. Papanikolaou N, Battista J, Mackie T, Kappas C, Boyer A. Tissue inhomogeneity corrections for megavoltage photon beams. AAPM Report No 85; Task Group No. 65, 2004.

3. Gray A, Oliver LD, Johnston PN. The accuracy of the pencil beam convolution and anisotropic analytical algorithms in predicting the dose effects due to attenuation from immobilization devices and large air gaps. Med Phys 2009; 36:3181-3191.

4. Kan MW, Cheung JY, Leung LH, Lau BM, Yu PK. The accuracy of dose calculations by anisotropic analytical algorithms for stereotactic radiotherapy in nasopharyngeal carcinoma. Phys Med Biol 2011; 56:397-413.

5. Martens C, Reynaert $\mathrm{N}$, de Wagter C, et al. Underdosage of the upper-airway mucosa for small fields as used in intensity-modulated radiation therapy: a comparison between radiochromic film measurements, Monte Carlo simulations, and collapsed cone convolution calculations. Med Phys 2002; 29:1528-1535.

6. Oyewale S. Dose prediction accuracy of collapsed cone convolution superposition algorithm in a multi-layer inhomogenous phantom. Int J Cancer Ther Oncol 2013; 1:01016.

7. Fogliata A, Vanetti E, Albers D, et al. On the dosimetric behaviour of photon dose calculation algorithms in the presence of simple geometric heterogeneities: comparison with Monte Carlo calculations. Phys Med Biol 2007; 52:1363-1385.

8. Rana S, Pokharel S. Verification of dose calculation algorithms in a multi-layer heterogeneous phantom using films. Gulf J Oncolog 2013; 1:63-69.

9. Robinson D. Inhomogeneity correction and the analytic anisotropic algorithm. J Appl Clin Med Phys 2008; 9:2786.

10. Rana SB. Dose prediction accuracy of anisotropic analytical algorithm and pencil beam convolution algorithm beyond high density heterogeneity interface. South Asian J Cancer 2013; 2:26-30.

11. Van Esch A, Tillikainen L, Pyykkonen J, Tenhunen M, Helminen H, Siljamaki S, Alakuijala J, Paiusco
M, Lori M, Huyskens DP. Testing of the analytical anisotropic algorithm for photon dose calculation. Med Phys 2006; 33:4130-4148.

12. Gagne IM, Zavgorodni S. Evaluation of the analytical anisotropic algorithm in an extreme water-lung interface phantom using Monte Carlo dose calculations. J Appl Clin Med Phys 2006; 8:33-46.

13. Sievinen J, Ulmer W, Kaissl W. AAA photon dose calculation model in Eclipse. Palo Alto (CA): Varian Medical Systems; 2005.

14. Ulmer W, Pyyry J, Kaissl W. A 3D photon superposition/convolution algorithm and its foundation on results of Monte Carlo calculations. Phys Med Biol 2005; 50:1767-1790.

15. Tillikainen L, Helminen H, Torsti T, Siljamäki S, Alakuijala J, Pyyry J, Ulmer W. A 3D pencil-beam-based superposition algorithm for photon dose calculation in heterogeneous media. Phys Med Biol 2008; 53:3821-3839.

16. Vassiliev ON, Wareing TA, McGhee J, Failla G, Salehpour MR, Mourtada F. Validation of a new grid-based Boltzmann equation solver for dose calculation in radiotherapy with photon beams. Phys Med Biol 2010; 55:581-598.

17. Bush K, Gagne IM, Zavgorodni S, Ansbacher W, Beckham W. Dosimetric validation of Acuros XB with Monte Carlo methods for photon dose calculations. Med Phys 2011; 38:2208-2221.

18. Han T, Mikell JK, Salehpour M, Mourtada F. Dosimetric comparison of Acuros XB deterministic radiation transport method with Monte Carlo and model-based convolution methods in heterogeneous media. MedPhys 2011; 38:2651-2664.

19. Kroon PS, Hol S, Essers M. Dosimetric accuracy and clinical quality of Acuros XB and AAA dose calculation algorithm for stereotactic and conventional lung volumetric modulated arc therapy plans. Radiat Oncol 2013; 8:149.

20. Kathirvel M, Subramanian S, Clivio A, Arun G, Fogliata A, Nicolini G, Subramani V, Thirumalai Swamy S, Vanetti E, Cozzi L. Critical appraisal of the accuracy of Acuros-XB and Anisotropic Analytical Algorithm compared to measurement and calculations with the compass system in the delivery of RapidArc clinical plans. Radiat Oncol 2013; 8:140.

21. Rana S, Rogers K. Dosimetric evaluation of Acuros $\mathrm{XB}$ dose calculation algorithm with measurements in predicting doses beyond different air gap thickness for smaller and larger field sizes. J Med Phys 2013; 38:9-14.

22. Rana S, Rogers K, Lee T, Reed D, Biggs C. Verification and dosimetric impact of Acuros XB algorithm for stereotactic body radiation therapy (SBRT) and RapidArc planning for non-small-cell lung cancer (NSCLC) patients. Int Jour of Med Phys Clin Eng Radiat Onc 2013; 2:6-14. 
23. Stathakis S, Esquivel C, Quino L, Myers P, Calvo O, Mavroidis P, Gutiérrez A and Papanikolaou N. Accuracy of the small field dosimetry using the Acuros $\mathrm{XB}$ dose calculation algorithm within and beyond heterogeneous media for $6 \mathrm{MV}$ photon beams. Int Jour of Med Phys Clin Eng Radiat Onc 2012; 1:78-87.

24. Dutreix J, Dutreix A, Tubiana M. Electronic equilibrium and transition stages. Phys Med Biol 1965; 10:177-190.

25. Das IJ, Ding GX, Ahnesjö A. Small fields: nonequilibrium radiation dosimetry. Med Phys 2008; 35:206-215.

26. Liu HW, Nugent Z, Clayton R, Dunscombe P, Lau $\mathrm{H}$, Khan R. Clinical impact of using the deterministic patient dose calculation algorithm Acuros XB for lung stereotactic body radiation therapy. Acta Oncol 2013. [Epub ahead of print]

27. Fogliata A, Nicolini G, Clivio A, Vanetti E, Cozzi L. Critical appraisal of Acuros XB and Anisotropic Analytic Algorithm dose calculation in advanced non-small-cell lung cancer treatments. Int J Radiat Oncol Biol Phys 2012; 83:1587-1595.

28. Khan RF, Villarreal-Barajas E, Lau H, Liu HW. Effect of Acuros XB algorithm on monitor units for stereotactic body radiotherapy planning of lung cancer. Med Dosim 2013. [Epub ahead of print]

29. Rana S, Rogers K, Pokharel S, Cheng C. Evaluation of Acuros XB algorithm based on RTOG 0813 dosimetric criteria for SBRT lung treatment with RapidArc. J Appl Clin Med Phys 2014; 15:4474.

30. Lu L. Dose calculation algorithms in external beam photon radiation therapy. Int J Cancer Ther Oncol 2013; 1:01025.

31. Fogliata A, Nicolini G, Clivio A, Vanetti E, Cozzi L. Dosimetric evaluation of Acuros XB advanced dose calculation algorithm in heterogeneous media. Radiat Oncol 2011; 6:82.
32. Han T, Followill D, Mikell J, Repchak R, Molineu A, Howell R, Salehpour M, Mourtada F. Dosimetric impact of Acuros $\mathrm{XB}$ deterministic radiation transport algorithm for heterogeneous dose calculation in lung cancer. Med Phys 2013; 40:051710.

33. Han T, Mourtada F, Kisling K, Mikell J, Followill D, Howell R. Experimental validation of deterministic Acuros XB algorithm for IMRT and VMAT dose calculations with the Radiological Physics Center's head and neck phantom. Med Phys 2012; 39:2193-2202.

34. Kan MW, Leung LH, So RW, Yu PK. Experimental verification of the Acuros XB and AAA dose calculation adjacent to heterogeneous media for IMRT and RapidArc of nasopharygeal carcinoma. Med Phys 2013; 40:031714.

35. Fogliata A, Scorsetti M, Navarria P, Catalano M, Clivio A, Cozzi L, Lobefalo F, Nicolini G, Palumbo V, Pellegrini C, Reggiori G, Roggio A, Vanetti E, Alongi F, Pentimalli S, Mancosu P. Dosimetric comparison between VMAT with different dose calculation algorithms and protons for soft-tissue sarcoma radiotherapy. Acta Oncol 2013; 52:545-552.

36. Dogan N, Siebers JV, Keall PJ. Clinical comparison of head and neck and prostate IMRT plans using absorbed dose to medium and absorbed dose to water. Phys Med Biol 2006; 51:4967-4980.

37. Keall P, Liu H. Dm rather than Dw should be used in Monte Carlo treatment planning. Med Phys 2002; 29:922-924. 